\title{
Cooperative awareness using roadside unit networks in mixed traffic
}

\author{
Manabu Tsukada*, Masahiro Kitazawa*, Takaharu Oi*, Hideya Ochiai*, and Hiroshi Esaki* \\ * Graduate School of Information Science and Technology, The University of Tokyo, Tokyo, Japan \\ Email: \{tsukada,ktzw,oi, jo2lxq\} @hongo.wide.ad.jp, hiroshi@wide.ad.jp
}

\begin{abstract}
Vehicle-to-vehicle (V2V) messaging is an indispensable component of connected autonomous vehicle systems. Although V2V standards have been specified by the European Union, United States, and Japan, the deployment phase represents mixed traffic in which connected and legacy vehicles coexist. To enhance cooperative awareness in this mixed traffic, we assessed the special roadside unit that we developed in our previous work that generates required $\mathrm{V} 2 \mathrm{~V}$ messages on behalf of sensed target vehicles. In this paper, we extend our earlier work to propose a system called Grid Proxy Cooperative Awareness Message to broaden the cooperative awareness message dissemination area by connecting infrastructure using high-speed roadside networks. To minimize delay in message delivery, we designed the proposed system to use edge computing. The proposed scheme delivers cooperative messages to a wider area with a low delay and a high packet delivery ratio by prioritizing packets by their respective safety contributions. Our simulation results indicate that the proposed scheme efficiently delivers messages in heavy road traffic conditions modeled on real maps of Tokyo and Paris.
\end{abstract}

Index Terms-vehicular networking, cooperative ITS, standards, cooperative awareness message, vehicle-to-vehicle

\section{INTRODUCTION}

Intelligent transportation systems (ITS) are being developed with the goal of making road traffic safer, more efficient, and more comfortable. Autonomous vehicles are currently attracting the interest of many researchers and engineers in the field of ITS. In 2018, Waymo (Google) initiated a taxi business using autonomous vehicles that navigate public roads in the United States, and similar attempts have followed worldwide. However, standalone autonomous vehicles as simple replacements of human driver operation (perception, decision, and maneuvering) share the driving limitations of humans. For example, such vehicles retain blind spots in their onboard sensors.

This limitation prevents autonomous vehicles from being safer and more efficient. For example, a standalone vehicle must stop at each intersection without a traffic signal before entering it slowly. As the risk of collision at an intersection depends on the time spent within the intersection, it would in theory be safer and more efficient to pass through the intersection at maximum speed. To achieve this, cooperative ITS can be applied to overcome vehicle blind spots by connecting the vehicle to roadside units.

The road network is inter-connected among countries, and there are few barriers and using the network, the vehicles easily cross the country border. For the interoperability among the countries, cooperative ITS needs to be developed based on the same architecture, protocols and technologies. To standardize cooperative ITS, the International Organization for Standardization (ISO) Technical Committee 204 Working Group 16 (TC204 WG16) (also known as Communications Architecture for Land Mobile (CALM)) is developing a standard architecture, called the ITS Station reference architecture, in coordination with the European Telecommunications Standards Institute (ETSI) TC ITS [1], [2]. In the United States, the Institute of Electrical and Electronics Engineers (IEEE) is standardizing a Wireless Access in Vehicular Environments (WAVE) architecture in the IEEE 1609 family of standards [3] and developing an IEEE802.11 variant for vehicular communication, IEEE802.11p [4].

Vehicle-to-vehicle (V2V) messaging is an indispensable cooperative ITS tool for achieving real-time information-sharing among vehicles. Using $\mathrm{V} 2 \mathrm{~V}$, vehicle positions and additional information can be communicated. The Cooperative Awareness Message (CAM) [5], Basic Safety Message (BSM) [6], and ITS forum basic messaging systems have been specified as V2V message standards in the EU, United States, and Japan, respectively.

Using V2V messaging, a connected autonomous vehicle can be made aware of other vehicles that are out of the line of sight. In the $\mathrm{V} 2 \mathrm{~V}$ deployment phase, however, it will be impossible to track the real-time information of all vehicles on the road owing to the presence of many legacy vehicles that do not have a transmitter. Therefore, mixed traffic in which connected autonomous vehicles and legacy vehicles coexist on the road must be assumed. In our previous work, we developed a special roadside unit called Proxy CAM agent [7] (also overviewed in Section II-B) to aid in the awareness of legacy vehicles. In that study, we implemented the agent and performed a field test at an intersection.

In this paper, we propose an inter-connection of Proxy CAM agents with high-speed roadside networks to enable the wider dissemination of cooperative awareness messages. The rest of the paper is organized as follows. Section II presents a problem statement of $\mathrm{V} 2 \mathrm{~V}$ messaging in mixed traffic and discusses potential solutions. Section III presents our proposal-Grid Proxy CAM-for enhancing cooperative awareness in mixed traffic using roadside unit networks. Section IV provides a numerical analysis to evaluate the proposed system. In Section $\mathrm{V}$, we evaluate the proposed system in a traffic simulation based on maps of Tokyo and Paris. In Section VI, we discuss 
related work and, finally, Section VII concludes the paper and outlines future related work.

\section{Problem Statement AND SOlUtion ANALYSiS}

\section{A. Issues in Mixed Traffic}

The penetration ratio of $\mathrm{V} 2 \mathrm{~V}$ devices is a critical factor in cooperative ITS because $\mathrm{V} 2 \mathrm{~V}$-based cooperative awareness does not work without $\mathrm{V} 2 \mathrm{~V}$ devices. The problem is that it is difficult to attain a sufficiently high penetration ratio in the deployment process because the traffic on the road will comprise a mixture of new and old vehicles. Accordingly, we need to consider mixed traffic of V2V-enabled and legacy vehicles. Aside from the issue of legacy vehicles, it is essential for cooperative ITS to accommodate pedestrians and bicycles, neither of which are usually equipped with V2V sender devices. Under the current $\mathrm{V} 2 \mathrm{~V}$ messaging paradigm, non$\mathrm{V} 2 \mathrm{~V}$ aware nodes are excluded and therefore the cooperative ITS network does not relay their presence to connected autonomous vehicles.

\section{B. Proxy Cooperative Awareness Message}

To make the presence of legacy vehicles available to connected autonomous vehicles, in our previous work we proposed Proxy CAM [7], an overview of which is shown in Fig. 1(a). Under Proxy CAM, roadside sensors detect target vehicles and estimate vehicle dynamic data such as positions, velocities, and accelerations. These vehicle data are sent from the sensors to an infrastructure-embedded server for storage in a database. The database then uses the stored data to generate $\mathrm{V} 2 \mathrm{~V}$ messages on behalf of the target vehicles as proxy $\mathrm{V} 2 \mathrm{~V}$ messages. The system uses CAMs to relay V2V information, although this could potentially be done using other protocols such as Basic Safety Messages (BSMs). Finally, the proxy CAMs are sent out by the roadside transmitters. The connected autonomous vehicles receive the proxy CAMs in the exact same manner that they would receive standard CAMs. As in Fig. 1(b), we implemented the Proxy CAM system using a stereo vision camera and a Linux OS wireless router. Then, we installed the system at an intersection in the campus of the University of Tokyo. The field test shows that the system successfully widens the dissemination area of the Proxy CAM.

\section{Objectives and solution analysis}

Using the Proxy CAM system, connected autonomous vehicles can be made aware of all of vehicles in mixed traffic. However, the message dissemination range remains limited to the range of wireless radio propagation from the roadside transmitters. Using IEEE802.11p in an ideal environment and over a line of sight, this range is generally from around 500 $\mathrm{m}$ to $1 \mathrm{~km}$, a distance that is often reduced in environments with non-line-of-sight scenarios.

The objective of the present study was to broaden the cooperative awareness message dissemination range by connecting Proxy CAM agents using a high-speed roadside network. We identified the following three requirements for designing a solution:

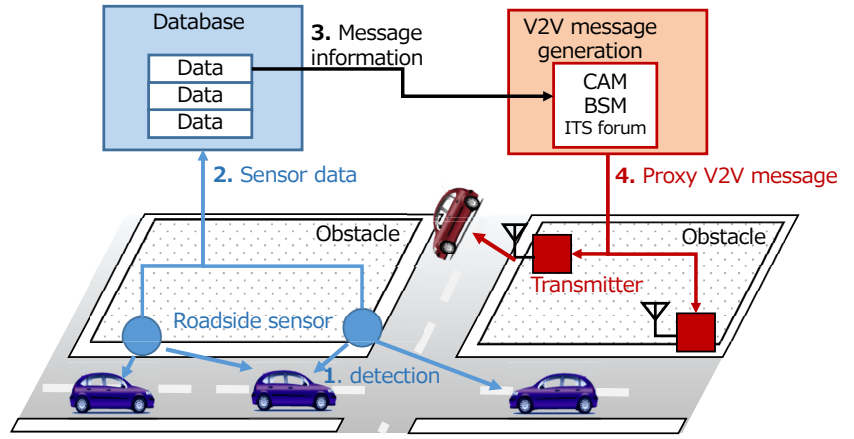

(a) Design of Proxy CAM

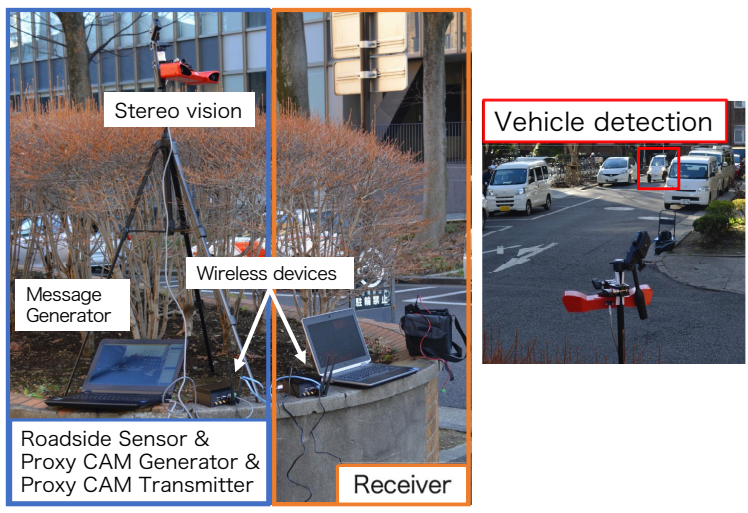

(b) Implementation of Proxy CAM

Fig. 1. Overview of Proxy CAM

- Coexistence with the CITS Standards: To enable interoperability among countries, cooperative ITS must be developed based on universal architecture, protocols, and technologies. Such a solution must adopt techniques for standardized V2V messaging such as CAM or BSM, as it should not have a large impact on current standards. In particular, CAM was specified in 2014 and is already used in many market-ready products.

- Real-time delivery of messages: Frequent transmission of $\mathrm{V} 2 \mathrm{~V}$ messages allows for the tracking of highly dynamic vehicle status information such as position, velocity, and acceleration. Standard CAMs, for example, are transmitted $1 \sim 10$ times per second. A solution should therefore be capable of frequent transmission of dynamic vehicle information. Overall, delays in message sensing and transmission must be minimized. The solution should be designed to deliver messages directly along the edge to bypass the Internet and the cloud systems.

- Message priority by safety contribution: The solution must frequently deliver messages over a broad area through a high-speed roadside network connection of Proxy CAM agents. The number of messages delivered will increase with road traffic. Along the message delivery path, the roadside wired network must have sufficient capacity to accommodate large numbers of messages at the peak road traffic condition. However, a wireless link will reach saturation if it receives too many messages. The 
solution must therefore prioritize messages in terms of importance based on their respective safety contributions.

\section{V2V MESSAGE DISSEMINATION USING ROADSIDE UNIT NETWORKS}

In this section, we propose a system that fulfills the three requirements listed in the previous section. The solution is divided into two components: a system (the Grid Proxy CAM) in which Proxy CAM agents are connected by roadside networks, and a distance priority algorithm to prioritize message delivery when road traffic is increased. The respective components are described in the following sections.

\section{A. Grid Proxy CAM}

Fig.2 shows an overview of the Grid Proxy CAM system. Each Proxy CAM agent comprises a sensor, transmitter, and router. The sensor and transmitter are connected to the router, which interconnects to all Proxy CAM agents via the wired network. An agent is installed at each intersection.

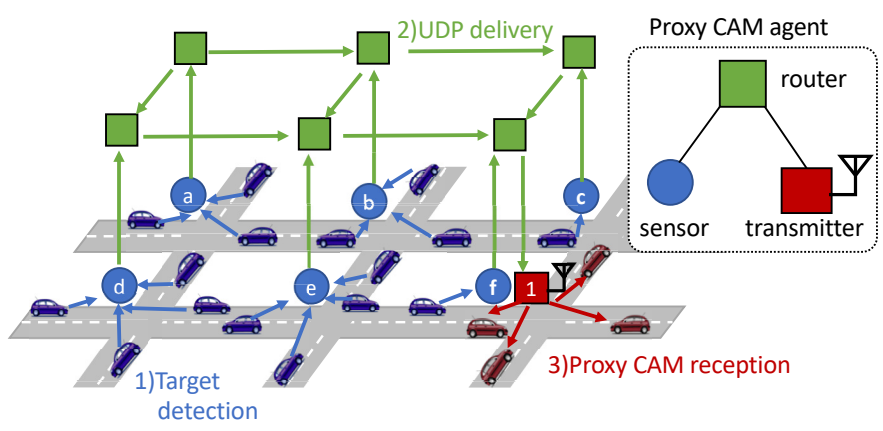

Fig. 2. Overview of Grid Proxy CAM system

As depicted in Fig.2 (1), the sensors (camera, LiDAR, or radar) can detect a target vehicle or pedestrian and estimate their position (latitude and longitude) and motion (speed and heading). Some sensors can also estimate object type (pedestrian, vehicle, bicycle, etc.) and vehicle class (public vehicle, emergency vehicle, etc.) if the data are available. If a number plate is visible, the sensors read the objects identification number; if not, a random number is assigned to the object and applied to the objects encoding within the sensor detection sequence.

As shown in Fig.2 (2), the estimated object information is sent via User Datagram Protocol (UDP) to all neighboring Proxy CAM agents within the range configured by the road infrastructure operator. It is assumed that each sensor has been provided in advance with a list of destination agent IP addresses. The (static or dynamic) IP routing system delivers the packet to its destination. The sensors send UDP packets at a rate of $10 \mathrm{~Hz}$, which is the maximum frequency under the CAM specification.

As shown in Fig.2(3), upon reception of the UDP packet the transmitter composes a proxy CAM in which unavailable object information is filled in as unknown. The transmitter then adds Basic Transport Protocol and GeoNetworking headers following the CAM standard specification. In preparation for broadcasting across the wireless link, the proxy CAM is added to the queue of the IEEE802.11p interface. The IEEE 802.11p MAC layer uses an Enhanced Distributed Channel Access(EDCA) [8] mechanism based on IEEE 802.11e with some parameter modifications; in the EDCA, the CAM is classified into the AC_BE class. The queuing priority policy is described in the next section.

The interconnected Proxy CAM agent operation described above makes real-time vehicle information available at all intersections. Fig. 2 shows an example in which vehicles (in red) receive a Proxy CAM of target vehicles (blue) via transmitter 1 from all of the neighboring sensors (a-f).

\section{B. Distance Priority algorithm}

A transmitter cannot broadcast all messages created when the target vehicle information increases significantly under heavy road traffic because the capacity of its wireless link will become saturated. The data rate of IEEE $802.11 \mathrm{p}$ is $3 \sim 27$ Mbps. The actual data-sending rate of proxy CAMs, however, is lower than the data rate because the message size is small, which increases the header ratio. When a transmitter receives more proxy CAMs than the sending rate, additional messages are added to the queue, increasing the wait time and end-to-end delay, which in turn makes real-time information outdated. For example, a sensor detecting vehicle moving at a speed of 50 $\mathrm{km} / \mathrm{h}$ requires $500 \mathrm{~ms}$ to complete a message delivery, during which time the difference between the position reported in the message and the actual position at the moment reaches approximately $7 \mathrm{~m}$. If the queue becomes full, messages must be discarded and the end-to-end packet delivery ratio (PDR) worsens.

Wider cooperative awareness incurs a trade-off in which a safer and more efficient ITS application is supported while the number of messages is increased, which in turn increases the delay and decreases the PDR. To make the proposed system practical, it is necessary to balance wider cooperative awareness, low delay, and high PDR. Our approach for carrying out this trade-off is to prioritize messages based on their safety contribution. We assume that the receiving node needs realtime information on the nearest objects for safety reasons-for example, to avoid crashes at an intersection-and prioritizes accordingly by applying a distance priority algorithm that gives higher priority to proxy CAMs from the transmitters that encode closer positions. The number of messages(i.e., number of detected objects), varies over time depending on the road traffic volume. If there is sufficient capacity to broadcast more proxy CAMs at a given moment, proxy CAMs with more distant object information are added to the queue.

The transmitter has a maximum distance of $d_{\max }$, which is pre-configured by the road infrastructure operator, and the queue occupancy ratio $r_{q o}$ is continuously monitored. Upon generation of a proxy CAM, the transmitter calculates the distance $d$ between position of the detected object in the message and the transmitter position to determine if the packet is to be added to the queue or dropped as follows: 


$$
r_{q o}<1-\frac{d}{d_{\max }} .
$$

If the equation returns true, the proxy CAM goes into the queue; otherwise, it is dropped. Using the equation, proxy CAMs that contain more distant object information are dropped when $r_{q o}$ becomes sufficiently large. The transmitter begins to drop the CAMs with more distant information before the queue is full but maintains room for nearer information for future use. The algorithm also reduces the value of $r_{q o}$, which helps to decrease the end-to-end delay.

\section{NUMERICAL ANALYSIS}

In this section, we conduct a mathematical analysis of the underlying performance of the proposed system in terms of message delivery delay and PDR.

We first calculate the effective throughput of the proxy CAMs over IEEE802.11p.

Table I summarizes the corresponding variables, symbols, and values used in the calculations. The average time interval needed to successfully transmit a CAM packet via the wireless channel, $t_{c a m}$, is given by

$$
t_{\text {cam }}=t_{D I F S}+b+t_{\text {send }},
$$

where $t_{D I F S}$ is the Distributed Inter Frame Space (DIFS) interval, $b$ is the backoff time, and $t_{\text {send }}$ is the duration of transmission.

TABLE I

PARAMETERS IN PHYSICAL AND MAC LAYERS

\begin{tabular}{rr|rr}
\hline Layer & Variable type & Symbol & Value \\
\hline \hline \multirow{3}{*}{ MAC } & AIFSN with OCB & $A I F S N$ & 6 \\
& Slot length of IEEE802.11p & slen $n_{11 p}$ & $0.013 \mathrm{~ms}$ \\
& SIFS interval & $t_{S I F S}$ & $0.032 \mathrm{~ms}$ \\
& Contention windows size & $C W$ & $0 \sim 15$ \\
& Average of $C W$ & $C W_{\text {ave }}$ & 7.5 \\
\hline \multirow{2}{*}{ PHY } & Switch time between Tx and Rx & $t_{\text {switch }}$ & $0.001 \mathrm{~ms}$ \\
& PLCP preamble duration & $t_{\text {pre }}$ & $0.032 \mathrm{~ms}$ \\
& Duration of PLCP Signal & $t_{\text {sig }}$ & $0.008 \mathrm{~ms}$ \\
& Symbol interval & $t_{\text {sym }}$ & $8 \mu \mathrm{s}$ \\
\hline Other & Payload Length of Proxy CAM & $P_{\text {pcam }}$ & $680 \mathrm{bits}$ \\
\hline
\end{tabular}

The DIFS interval $t_{D I F S}$ is the waiting time after the channel becomes an idle state in CSMA/CA and is given by

$$
t_{D I F S}=A I F S N \times \text { slen }_{11 p}+t_{S I F S} .
$$

For the AC_BE class in EDCA, the arbitration interframe space number (AIFSN) $A I F S N$ when Offset Codebook Mode (OCB) is activated is six [9]. The slot length of IEEE801.11p slen $_{11 p}=0.013 \mathrm{~ms}$ and the Short Inter Frame Space (SIFS) interval $t_{S I F S}=0.032 \mathrm{~ms}$; therefore, from equation $3, t_{D I F S}$ is $0.110 \mathrm{~ms}$.

The backoff time $b$ is the random waiting time determined by the contention window and is given by

$$
b=C W_{\text {ave }} \times \text { slen }_{11 p}
$$

where the contention window size, $C W$, is $0 \sim 15$ [9]. The average of the contention windows size $C V_{\text {ave }}=7.5$; thus, from equation $4, b=0.0975 \mathrm{~ms}$.

The transmission duration, $t_{\text {send }}$, of a proxy CAM is given by

$$
\begin{aligned}
t_{\text {send }}= & t_{\text {switch }}+t_{\text {pre }}+t_{\text {sig }} \\
& +t_{\text {sym }} \times \operatorname{ceil}\left(\frac{16+P_{\text {pcam }}+6}{N_{D B P S}}\right),
\end{aligned}
$$

where $t_{\text {switch }}$ is the switch time between $\mathrm{Tx}$ and $\mathrm{Rx}, t_{\text {pre }}$ is the duration of the Physical Layer Convergence Protocol (PLCP) preamble, $t_{s i g}$ is the duration of the PLCP signal, $N_{D B P S}$ is the number of data bits per OFDM symbol, $t_{\text {sym }}$ is the symbol interval, and $\operatorname{ceil}()$ is a function returning the smallest integer greater than or equal to a given number. In IEEE802.11p $t_{\text {switch }}=0.001 \mathrm{~ms}, t_{\text {pre }}=0.032 \mathrm{~ms}$, and $t_{\text {pre }}=$ $0.008 \mathrm{~ms}$. For a data rate of $18 \mathrm{Mbps}$, IEEE802.11p specifies the use of 16QAM for the modulation scheme and a code rate fixed at $3 / 4$. Therefore, $N_{D B P S}=144$ bits and $t_{\text {sym }}=8 \mu \mathrm{s}$, making $t_{\text {send }}=0.081 \mathrm{~ms}$ from equation 5 .

From equation 2, the average time interval needed to successfully transmit a CAM packet is $t_{\text {cam }}=0.2885 \mathrm{~ms}$. A proxy CAM (payload length $P_{\text {pcam }}=680$ bits) is transmitted over $0.2885 \mathrm{~ms}$ on average, resulting in an effective proxy CAM throughput over IEEE802.11p of about 2.73 Mbps.

From this, we can calculate the data rate at which the transmitter can broadcast CAMs without having to retain any in the queue: a broadcast rate of 273 kbits in $0.1 \mathrm{~s}$ at an effective throughput of $2.73 \mathrm{Mbps}$ equates to a transmission of 401 CAMs every $0.1 \mathrm{~s}$. If each proxy CAM agent detects ten vehicles on average, it can broadcast messages from approximately 40 neighboring agents. In other words, queuing will begin when the agent detects more than ten vehicles or when more than 40 neighboring agents send messages. Thus, the end-to-end delay increases while the end-to-end PDR decreases in situations in which the distance priority algorithm is not applied. In the case of a 1,000-packet queue length, which is applied in the simulation scenario in Section V), the queuing delay is $0.2885 \mathrm{~ms} \times 1000=288.5 \mathrm{~ms}$ when the queue is full.

\section{Evaluation}

\section{A. Simulation Scenarios}

1) Environment: To evaluate the communication performance in terms of delay and PDR under various scenarios, we implemented our proposed method on the Artery ${ }^{1}$ framework, an extension of the open source vehicular network simulation framework Veins ${ }^{2}$. In Veins, the communication network is simulated in $O M N e T+{ }^{3}$ while vehicle traffic (i.e., movement) is simulated in $S U M O^{4}$.

\footnotetext{
${ }^{1}$ https://github.com/riebl/artery

${ }^{2}$ http://veins.car2x.org

${ }^{3}$ https://omnetpp.org

${ }^{4}$ http://sumo.dlr.de/
} 
2) Maps: We conducted experiments using maps of Tokyo, Japan, and Paris, France. Figs 3 and 4 show the maps, which cover the area near the University of Tokyo and the city of Paris, respectively. Both maps were taken from the OpenStreetMap wiki. The size of the Tokyo map is about $1.9 \mathrm{~km} \times 1.7 \mathrm{~km}$, while that of the Paris map is around $1.8 \mathrm{~km} \times 2.0 \mathrm{~km}$. Each selected intersection has a proxy CAM agent and all agents are interconnected by a 10-Gbps Ethernet cable (black line). The Tokyo and Paris maps have 49 and 32 agents, respectively. Each agent maintains the routes to the other agents using the Routing Information Protocol (RIP). The red lines in the maps indicate buildings (obstacles) that attenuate wireless radio when blocking the line of sight between a source and destination node. Each building edge blocking a path attenuates the signal by $9 \mathrm{db}$; a signal passing through a building is attenuated by $0.4 \mathrm{db}$ per meter.

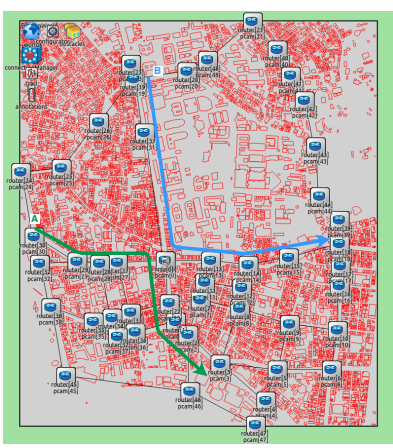

Fig. 3. Map of Tokyo

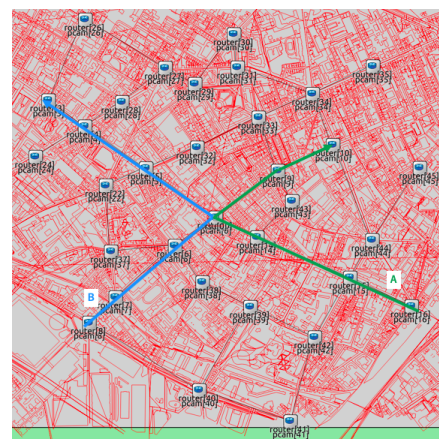

Fig. 4. Map of Paris
3) Scenario: We conducted and compared the results of experiments covering the following two scenarios: 1) use of a Grid Proxy CAM algorithm, and 2) use of a Grid Proxy $\mathrm{CAM}+$ distance priority algorithm. The parameters of the simulations are listed in Table II.

TABLE II

PARAMETERS OF SimUlations

\begin{tabular}{c|lc}
\hline Type & Variable name & Value \\
\hline \hline & IEEE802.11p datarate & $18 \mathrm{Mbps}$ \\
Radio & Attenuation per building edge & $9 \mathrm{db}$ \\
& Attenuation through building & $0.4 \mathrm{db} / \mathrm{m}$ \\
& Radio range & $150 \mathrm{~m}$ \\
\hline Proxy & Queue length & $1000 \mathrm{packets}$ \\
CAM & Maximum distance $\left(d_{\max }\right)$ & $1000 \mathrm{~m}$ \\
agent & Proxy CAM frequency & $10 \mathrm{~Hz}$ \\
\hline Vehicle & Vehicle speed & $50 \mathrm{~km} / \mathrm{h}$ \\
traffic & Vehicle num per sec per intersection & 2 \\
& CAM frequency & $10 \mathrm{~Hz}$ \\
\hline
\end{tabular}

The IEEE $802.11 \mathrm{p}$ data rate, radio frequency, transmission power, and reception sensitivity were $18 \mathrm{Mbps}, 5.89 \mathrm{GHz}, 126$ $\mathrm{mW}$, and $-89 \mathrm{dBm}$, respectively. We limited the maximum radio range to $150 \mathrm{~m}$ to reflect the typical radio coverage limitations within an urban area. Each proxy CAM agent generated messages at a frequency of $10 \mathrm{~Hz}$. The maximum distance $\left(d_{\max }\right.$, as defined in Section III), was $1,000 \mathrm{~m}$ and the queue length was 1,000 packets.
The simulated vehicles traveled at $50 \mathrm{~km} / \mathrm{h}$ through intersections along two routes, (A) and (B), which are marked in Figs 3 and 4. Each vehicle passed through intersections without stopping, as we assumed that the roads had no traffic signals; two vehicles per second passed through each intersection. The vehicle traffic through the specified intersections of the Tokyo map (Fig 3) was the average traffic reported in the official traffic statistics of the Japanese Police department. The vehicles generated standard CAMs at a frequency of $10 \mathrm{~Hz}$.

Each agent detected five, ten, or fifteen vehicles every $0.1 \mathrm{~s}$ and sent UDPs to all neighboring agents. Assuming a sensor detection coverage of $50 \mathrm{~m}$, each sensor covered a total of 200 $\mathrm{m}$ in the four directions pointing outward from its intersection. We therefore set the traffic rate in the respective scenarios at 25,50 , and 75 vehicles $/ \mathrm{km}$ to reflect the detection of five, ten, and fifteen vehicles per $200 \mathrm{~m}$, respectively.

As shown in Fig 5, we measured the communication performance at the intersections at which the two routes crossed (located at the centers of the maps). To obtain measurements, we used an evaluation node installed close to the intersection to receive proxy CAMs via IEEE802.11p from all neighbouring agents. We evaluated the packet delivery ratio and the delay from the source agent to the evaluation node. We conducted all simulations 100 times each with random seeds and calculated the averages and standard deviations for the results. Each simulation lasted $15 \mathrm{~s}$, with the results from $5-10 \mathrm{~s}$ used for evaluation to ensure that communication performance was measured in the steady state.

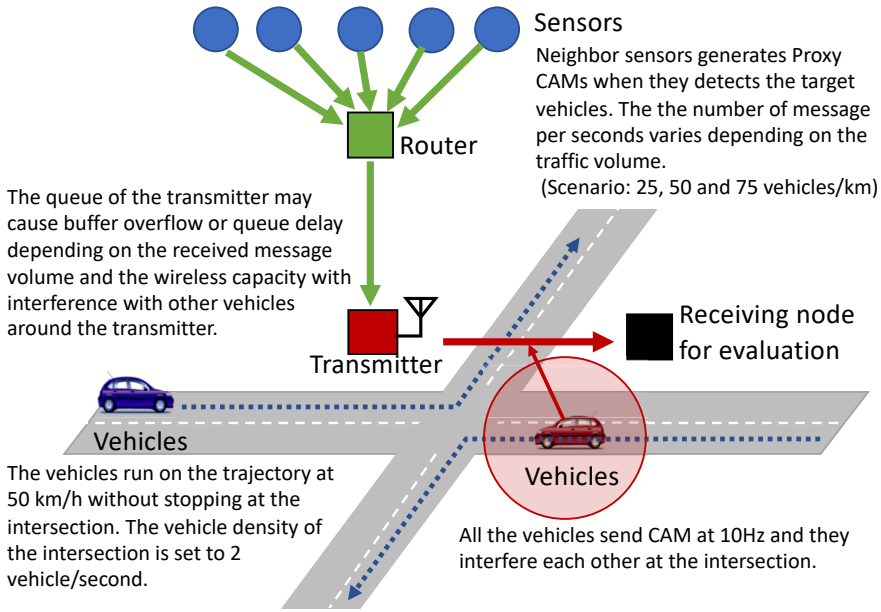

Fig. 5. Simulation Scenarios

\section{B. Packet delivery ratio evaluation}

Fig 6 shows the PDR of the Tokyo map simulations with vehicle densities of 25,50 , and 75 vehicles $/ \mathrm{km}$. The red and blue lines show the Grid Proxy CAM scenarios without and with the distance priority algorithm, respectively. Both lines indicate the average PDRs, with the colored zones above and below showing the standard deviations. As expected, both Grid Proxy CAM schemes could deliver messages over longer 

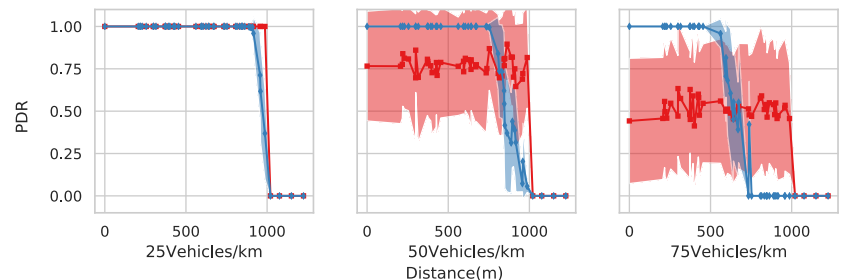

- Grid Proxy CAM

Fig. 6. PDRs of Tokyo scenarios
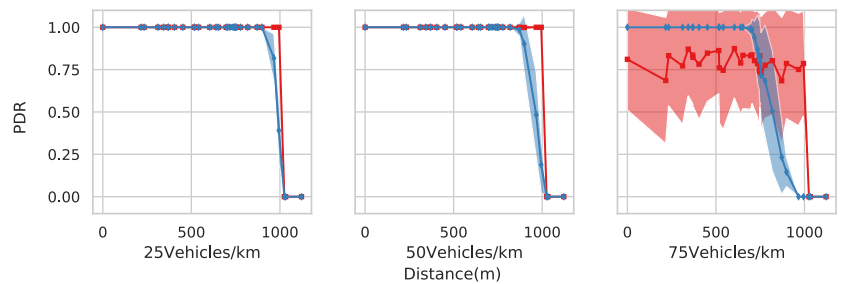

F- Grid Proxy CAM

Fig. 7. PDRs of Paris scenario

distances than the Proxy CAM scenario, in which no packet was delivered further than the wireless range $(150 \mathrm{~m})$ under any of the vehicle density scenarios.

At a vehicle density of 25 vehicles/km, the Grid Proxy CAM scenario maintained a PDR of $100 \%$ at all distances. By contrast, the Grid Proxy CAM with distance priority algorithm scenario maintained a $100 \%$ PDR up to $900 \mathrm{~m}$, after which it gradually dropped up to $1,000 \mathrm{~m}$. The drop occurred because the algorithm discarded more distant information to maintain room for closer information.

At a vehicle density of 50 vehicles $/ \mathrm{km}$, the PDR of the Grid Proxy CAM scenario was maintained at around $70 \%$ with large standard deviations, which arose for the following reasons. The first-arriving packets at the agent in question were successfully transmitted to the receiving node, but succeeding packets risked packet drop depending on the queue occupancy ratio. As the simulation reflected the randomness of vehicle detection timing, packets from multiple distances were targeted by the packet drop, which kept the standard deviation high. Applying the distance priority algorithm, by contrast, resulted in a PDR of $100 \%$ within a distance of $740 \mathrm{~m}$, after which the PDR gradually dropped up to a distance of $990 \mathrm{~m}$; in this scenario, the randomness of the detection timing also resulted in a relatively large standard deviation.

At a vehicle density of 75 vehicles $/ \mathrm{km}$, under the Grid Proxy CAM scenario, half of the messages were lost at all distances. Applying the distance priority algorithm, however, enabled the delivery of $100 \%$ of the messages within a range of $450 \mathrm{~m}$, with the PDR gradually dropping thereafter up to $800 \mathrm{~m}$.

Fig.7 shows the PDRs of the Paris map, At vehicle densities of 25 and 50 vehicles $/ \mathrm{km}$, the PDR is $100 \%$ in the nopriority scenario at all distances because there are fewer agents installed in the selected intersection. The distance priority algorithm drops messages from longer distances (900 and $850 \mathrm{~m}$ ) to enable the reservation of closer information. At 75 vehicles $/ \mathrm{km}$, the distance priority algorithm maintains a PDR of $100 \%$ within $650 \mathrm{~m}$, whole the no-priority scenario is unstable at all distances.

As the above results show, using Grid Proxy CAM widens the message transmission range and enables the priority algorithm to sustain a $100 \%$ message delivery rate for closer information.

\section{Delay evaluation}

Fig 8 shows the delays in the Tokyo simulation at vehicle densities of 25,50 , and 75 vehicles $/ \mathrm{km}$. At 25 vehicles $/ \mathrm{km}$, the delay is approximately $10 \mathrm{~ms}$ under both Grid Proxy CAM scenarios up to the maximum distance of $1,000 \mathrm{~m}$. At 50 vehicles $/ \mathrm{km}$, the no-priority scenario has a $290-\mathrm{ms}$ delay caused by the queuing delay calculated in Section IV. The distance priority algorithm reduces the delay to $40 \mathrm{~ms}$ within $740 \mathrm{~m}$, in which the PDR is $100 \%$. The delay then decreases from 740 to $990 \mathrm{~m}$ because the packet occupancy ratio decreases over this range (i.e., packet drop occurs). At 75 vehicles $/ \mathrm{km}$, the Grid Proxy CAM scenario has a delay of $290 \mathrm{~ms}$. The distance priority algorithm reduces the delay to $100 \mathrm{~ms}$ within $450 \mathrm{~m}$, in which the PDR is $100 \%$. For the reason mentioned above, the delay decreases from 450 to 800 $\mathrm{m}$.

Fig 9 shows the delays in the Paris map simulations. At 25 vehicles $/ \mathrm{km}$, the delay is around $10 \mathrm{~ms}$ under both scenarios. The delay increases to about $20 \mathrm{~ms}$ at 50 vehicles $/ \mathrm{km}$ under both scenarios. At 75 vehicles $/ \mathrm{km}$, the delay is around 290 ms without the proposed algorithm, while the distance priority algorithm reduces the delay to $65 \mathrm{~ms}$.

The above results indicate that the Grid Proxy CAM system can deliver messages widely and that the distance priority algorithm contributes to reducing the delay for closer information.

\section{RELATED WORKS}

As an infrastructure assisted cooperative ITS, the Japan Metropolitan Police Department develops Driving Safety Support Systems (DSSS) for accident reduction [10]. DSSS experimented three systems: rear-end collision prevention system at entering to traffic jam which hides behind obstacles, collision prevention system at turning right at the intersection, and collision prevention system at the intersection in a bad view. The infrastructure assisted CITS using beacons and FM broadcasting are implemented on the highways in Japan.

It is analyzed that performance of Vehicular Ad-Hoc Network (VANET) depends on TX power, frequency of transmission, and the lifetime of messages in $\mathrm{V} 2 \mathrm{~V}$ communications and V2I communications [11]. Furthermore, it is known that performance of $\mathrm{V} 2 \mathrm{~V}$ messages and V2I messages strongly depends on the link quality and the propagation conditions [12]. [12] demonstrates that awareness levels for V2I communications are better than $\mathrm{V} 2 \mathrm{~V}$ communications if the roadside units are implemented on advantageous positions. [12] also 


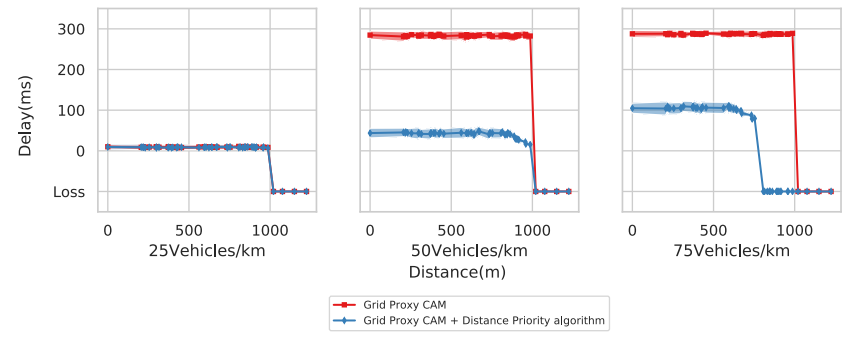

Fig. 8. Delays under Tokyo scenarios
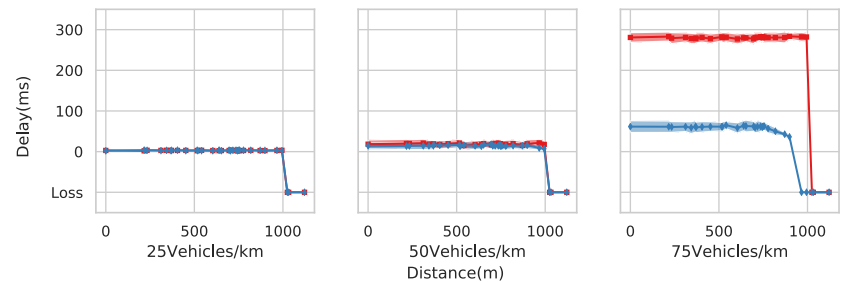

- Grid proxy CAM

Fig. 9. Delays under Paris scenarios

explains that TX power is more important than frequency of transmission for V2X communications.

There are many ways of sensing which are useful for road traffic. Vision-based vehicle detection and tracking techniques are summarized in [13]. In vision-based technics, Millimeterwave radar and a camera are important equipment for sensing vehicles. Millimeter-wave radar can be used for measuring target range and speed. Police use Millimeter-wave radar in traffic speed regulation [13]. Millimeter-wave radar is available with a poor view in a bad weather. Stereo cameras are very effective way to sensing vehicles. Stereo cameras can detect a vehicle and, sense vehicle's position and velocity [14].

In our previous work [15], we presented a detailed problem statement on the mixed environment and a requirements analysis of infrastructure-assisted messaging. [16] extended the Proxy CAM framework to delivery to remote places via the Internet using IPv6 and LTE.

Several approaches have been developed to create messages for sharing the perception objects by sensors. Cooperative Perception Messages (CPM) is specified in Ko-PER as a method for perceiving dynamic objects in the environment of an equipped vehicle or roadside station [17], [18]. Environmental Perception Message (EPM) is a proprietary message framework that includes a list of all perceived objects containing the unique IDs of all vehicles perceived by local perception sensors [19], [20]. Sensory Observation Message (SOM) has been proposed as a method for sharing infrastructure sensor information primarily for the purpose of defending vulnerable road users [21]. [22] V2X-based cooperative protection systemfor vulnerable road users and its impact on traffic

Cloud-based cooperative awareness between vehicles and pedestrians was proposed in [23]. Under this framework, pedestrians send their positions to the cloud regularly from their smartphones so that the cloud can alert vehicles to approaching pedestrians. Vehicle-to-Pedestrian (V2P) communication was investigated in [24], who developed a system in which pedestrians receive CAMs from invisible vehicles from behind an obstacle on their smartphones.

[25] demonstrated how multi-sensor data fusion can leverage consistency and plausibility checking of perception sensor data. In particular, it validated the contents of CAMs delivered by connected vehicles using independent on-board perception sensors.

In the research domain of cooperative autonomous driving, [26] introduce a method of occupancy grid map merging, dedicated to multivehicle cooperative local mapping purpose in outdoor environments. [27], [28] propose a multimodal cooperative perception system that provides see-through, liftedseat, satellite and all-around views to drivers. The features are validated in real-world experiments using four vehicles on the road.

For the traffic management in the intersection, traffic lights are installed to coordinate the traffic flows. In the connected autonomous vehicle age, [29] proposes to introduce autonomous intersection management. In the proposal, vehicles coming to an intersection connect to a dedicated intersection controller for the reservation. [30] extends the prioritybased coordination approach at an intersection to support autonomous and legacy vehicles.

\section{CONCLUSION AND FUTURE WORK}

In this paper, we proposed the Grid Proxy CAM system, in which high-speed roadside networks of interconnected roadside sensors are used to widen the dissemination of proxy cooperative awareness messages. To enable the system to function under conditions of high message traffic, we proposed a distance priority algorithm that gives higher priority to proxy CAMs corresponding to positions closer to the receiver. Our simulation results demonstrate that the proposed scheme successfully delivers messages with low latency and at a high delivery ratio, particularly under heavy road traffic conditions.

The future work includes adaptive maximum distance. In this study we set the maximum distance $d_{\max }$, as preconfigured by the road infrastructure operator, as a constant $(1,000 \mathrm{~m})$. In theory, it should be possible to introduce an adaptive parameter to the maximum distance to cover additional area when the road traffic is low. To achieve an adaptive maximum distance, the queue monitoring results must be shared among neighboring agents to ensure that the proper number of messages is sent to each agent.

\section{ACKNOWLEDGMENT}

This work was partly supported by JSPS KAKENHI Grant Number JP17H04678.

\section{REFERENCES}

[1] ISO 21217:2010 Intelligent transport systems - Communications access for land mobiles (CALM) - Architecture, April 2010 
[2] Intelligent Transport Systems (ITS); Communications Architecture, September 2010. ETSI EN 302665 V1.1.1 (2010-09).

[3] IIEEE Guide for Wireless Access in Vehicular Environments (WAVE) Architecture, April 2013. EEE 1609.0-2013.

[4] IEEE Standard for Information technology - Telecommunications and information exchange between systems - Local and metropolitan area networks - Specific requirement, Part 11: Wireless LAN Medium Access Control (MAC) and Physical Layer (PHY) Specifications, July 2010. IEEE Std 802.11p-2010.

[5] Intelligent Transport Systems (ITS); Vehicular Communications; Basic Set of Applications; Part 2: Specification of Cooperative Awareness Basic Service, December 2014. ETSI EN 302 637-2 V1.3.2 (2014-11).

[6] SAE J2735 Dedicated Short Range Communications (DSRC) Message Set Dictionary, November 2009.

[7] T. Kitazato, M. Tsukada, H. Ochiai, and H. Esaki. Proxy cooperative awareness message: an infrastructure-assisted v2v messaging. In 2016 Ninth International Conference on Mobile Computing and Ubiquitous Networking (ICMU), pages 1-6, Oct 2016.

[8] IEEE standard for information technology-local and metropolitan area networks-specific requirements-part 11: Wireless LAN medium access control (MAC) and physical layer (PHY) specifications - amendment 8: Medium access control (MAC) quality of service enhancements. IEEE Std 802.11e-2005 (Amendment to IEEE Std 802.11 Edition (Reaff 2003), pages 1-212, November 2005.

[9] IEEE standard for information technology-telecommunications and information exchange between systems local and metropolitan area networks-specific requirements part 11: Wireless LAN medium access control (MAC) and physical layer (PHY) specifications. IEEE Std 802.11-2012 (Revision of IEEE Std 802.11-2007), pages 1-2793, March 2012.

[10] Nakagawa Atsushi, Nakano Tsuyoshi, and Okamoto Yasukazu. Demonstration Experiments of Driving Safety Support Systems Using Vehicleto-Infrastructure Communications Systems (Japanese), 2009.

[11] Martijn Van Eenennaam, Wouter Klein Wolterink, Georgios Karagiannis, and Geert Heijenk. Exploring the solution space of beaconing in vanets. In Vehicular Networking Conference (VNC), 2009 IEEE, pages 1-8. IEEE, 2009.

[12] Mate Boban and Pedro M. d'Orey. Exploring the practical limits of cooperative awareness in vehicular communications. March 2015.

[13] S. Sivaraman and M. M. Trivedi. Looking at vehicles on the road: A survey of vision-based vehicle detection, tracking, and behavior analysis. 14(4):1773-1795, December 2013.

[14] M. Bertozzi, A. Broggi, A. Fascioli, and S. Nichele. Stereo visionbased vehicle detection. In Intelligent Vehicles Symposium, 2000. IV 2000. Proceedings of the IEEE, pages 39-44, 2000.

[15] Manabu Tsukada. Roadside-assisted $\mathrm{v} 2 \mathrm{v}$ messaging for connected autonomous vehicle. In The Thirteenth International Conference on Wireless and Mobile Communications (ICWMC 2017), pages 89-94, Nice, France, 2017.

[16] Masahiro Kitazawa, Manabu Tsukada, Kei Morino, Hideya Ochiai, and Hiroshi Esaki. Remote Proxy V2V Messaging using IPv6 and GeoNetworking. In Vehicular 2017, July 2017.

[17] Andreas Rauch, Felix Klanner, and Klaus Dietmayer. Analysis of v2x communication parameters for the development of a fusion architecture for cooperative perception systems. In Intelligent Vehicles Symposium (IV), 2011 IEEE, pages 685-690. IEEE, 2011.

[18] Andreas Rauch, Stefan Maier, Felix Klanner, and Klaus Dietmayer. Inter-vehicle object association for cooperative perception systems. In Intelligent Transportation Systems-(ITSC), 2013 16th International IEEE Conference on, pages 893-898. IEEE, 2013.

[19] Hendrik-jorn Gunther, Oliver Trauer, and Lars Wolf. The potential of collective perception in vehicular ad-hoc networks. In ITS Telecommunications (ITST), 2015 14th International Conference on, pages 1-5. IEEE, 2015.

[20] Hendrik-Jörn Günther, Björn Mennenga, Oliver Trauer, Raphael Riebl, and Lars Wolf. Realizing collective perception in a vehicle. In Vehicular Networking Conference (VNC), 2016 IEEE, pages 1-8. IEEE, 2016.

[21] Robbin Blokpoel and Arjan Stuiver. Sensory observation message and cam extensions for vru safety.

[22] Tim Ruß, Jan Krause, and René Schönrock. V2x-based cooperative protection system for vulnerable road users and its impact on traffic.

[23] M. Bagheri, M. Siekkinen, and J. K. Nurminen. Cellular-based vehicle to pedestrian (v2p) adaptive communication for collision avoidance. In
Connected Vehicles and Expo (ICCVE), 2014 International Conference on, pages 450-456, November 2014.

[24] P. Merdrignac, O. Shagdar, I. B. Jemaa, and F. Nashashibi. Study on perception and communication systems for safety of vulnerable road users. In Intelligent Transportation Systems (ITSC), 2015 IEEE 18th International Conference on, pages 1876-1881, September 2015.

[25] Marcus Obst, Laurens Hobert, and Pierre Reisdorf. Multi-sensor data fusion for checking plausibility of $\mathrm{v} 2 \mathrm{v}$ communications by vision-based multiple-object tracking. In Vehicular Networking Conference (VNC), 2014 IEEE, pages 143-150. IEEE, 2014.

[26] Hao Li, M Tsukada, F Nashashibi, and M Parent. Multivehicle Cooperative Local Mapping: A Methodology Based on Occupancy Grid Map Merging. Intelligent Transportation Systems, IEEE Transactions on, 15(5):2089-2100, October 2014.

[27] Seong-Woo Kim, Zhuang Jie Chong, Baoxing Qin, Xiaotong Shen, Zhuoqi Cheng, Wei Liu, and Marcelo H Ang. Cooperative perception for autonomous vehicle control on the road: Motivation and experimental results. In Intelligent Robots and Systems (IROS), 2013 IEEE/RSJ International Conference on, pages 5059-5066. IEEE, 2013.

[28] Seong-Woo Kim, Baoxing Qin, Zhuang Jie Chong, Xiaotong Shen, Wei Liu, Marcelo H Ang, Emilio Frazzoli, and Daniela Rus. Multivehicle cooperative driving using cooperative perception: Design and experimental validation. IEEE Transactions on Intelligent Transportation Systems, 16(2):663-680, 2015.

[29] Kurt Dresner and Peter Stone. A multiagent approach to autonomous intersection management. Journal of artificial intelligence research, 31:591-656, 2008.

[30] Xiangjun Qian, Jean Gregoire, Fabien Moutarde, and Arnaud De La Fortelle. Priority-based coordination of autonomous and legacy vehicles at intersection. In Intelligent Transportation Systems (ITSC), 2014 IEEE 17th International Conference on, pages 1166-1171. IEEE, 2014. 